\title{
ESTUDIO DE UN NUEVO PROCEDIMIENTO PARA MEDIR LA CONDUCTIVIDAD Y DIFUSIVIDAD TÉRMICAS DE MATERIALES
}

\section{STUDY OF A NEW APPROACH FOR MEASURING THERMAL CONDUCTIVITY AND DIFFUSIVITY OF MATERIALS}

\author{
Rodney Hechavarría ${ }^{1, *}$, Osvaldo Delgado ${ }^{2}$, Francisco Pazmiño ${ }^{3}$
}

\section{Resumen}

En este trabajo se estudia las posibilidades y limitaciones de un método no-estacionario, que utiliza como fuente de calor la radiación luminosa. En el mismo, que es una nueva variante propuesta por este grupo, la luz se hace incidir homogéneamente sobre una de las superficies extremas de un cilindro de superficie lateral termoaislada, manteniéndose constante la temperatura en el otro extremo. Asimismo, se analiza la posibilidad de utilizar dicho método para medir la difusividad y conductividad térmicas $(\alpha, \chi)$ de fluidos; igualmente, la forma en que este pudiera ser empleado para comprobar la validez del modelo de Hamilton y Crosser (HC) para el caso de los nanofluidos. Se le llama nanofluido a todo aquel fluido que, convencionalmente, es utilizado para intercambiar calor, al cual se le agregan nanopartículas con el propósito de aumentar su conductividad térmica, mejorando así su capacidad para intercambiar calor.

Palabras clave: conductividad térmica, difusividad térmica, conducción de calor, modelo de Hamilton y Crosser.

\begin{abstract}
In the present work it has been studied the possibilities and limitations of non-stationary method, which uses heat as a source of light radiation. In this method, a new variant proposed by this group, the light is impinged evenly over one of the end surfaces of a cylinder lateral surface heat insulated, keeping the temperature constant at the other end. Similarly, the possibility of using the above method for measuring the diffusivity and thermal conductivity $(\alpha, \chi)$ fluid is analyzed and how it could be used to check the validity of the Hamilton and Crosser's model (HC) to the case of nano-fluids. It has been called nano-fluid, fluid anyone conventionally used for heat exchange, to which are added nano-particles in order to increase its thermal conductivity, thereby improving their ability to exchange heat.
\end{abstract}

Keywords: thermal conductivity, thermal diffusivity, heat conduction, Hamilton and Crosser model.

\footnotetext{
${ }^{1, *}$ Facultad de Ingeniería Civil y Mecánica, Universidad Técnica de Ambato - Ecuador, Autor para correspondencia : uronny2@yahoo.com

${ }^{2}$ Facultad de Ingeniería en Energía, Universidad Politécnica Metropolitana de Hidalgo - México.

${ }^{3}$ Facultad de Ingeniería Civil y Mecánica, Universidad Técnica de Ambato - Ecuador

Recibido: 30-09-2016, aprobado tras revisión: 02-12-2016

Forma sugerida de citación: Hechavarría, R.; Delgado, O.; Pazmiño, F (2017). «Estudio de un nuevo procedimiento para medir la conductividad y difusividad térmicas de materiales». INGENIUS. N. ${ }^{\circ} 17$, (Enero-Junio). pp. 15-22. ISSN: 1390-650X.
} 


\section{Introducción}

En la actualidad se conocen varios métodos que permiten determinar la conductividad y difusividad térmicas de los materiales [1-8], [9-13]. Por eso, la elección correcta del método apropiado en cada situación particular es de gran importancia. Para hacerlo es necesario tomar en cuenta diversos factores.

1. Si no se requiere de una gran precisión, entonces son innecesarios los métodos muy sofisticados.

2. Los métodos estacionarios son mayormente de larga duración y por ello no son convenientes para medir un gran número de muestras.

3. Si no es posible preparar muestras de geometría definida, se deben preferir los métodos que empleen fuentes puntuales y lineales.

Todos los métodos utilizados para medir propiedades térmicas se pueden dividir en dos grupos fundamentales: $I$ ) los métodos que utilizan fuentes de calor y $I I$ ) aquellos que prescinden de estas. En el último grupo la temperatura $T$ del medio se modula por contacto con un intercambiador de calor infinito, mientras que en el primero la fuente de calor actúa en el interior de la muestra o en su superficie.

Los métodos que no utilizan fuentes de calor se pueden dividir a su vez en estacionarios y en noestacionarios. Por su parte, los métodos que usan fuentes de calor también se dividen en estacionarios y no-estacionarios; pero, en este caso, otros factores de clasificación pasan a ser más importantes, a saber: a) la geometría de la fuente (puntual, lineal, superficial o volumétrica), b) la dependencia temporal de la fuente de potencia (régimen de pulso o continuo) y c) la configuración de la muestra que se mide (ejemplo, plano-paralela, cilíndrica o esférica). Puesto que, cada instalación experimental puede emplear varias combinaciones de los tres grupos $a)-c$ ) existe un gran número de modificaciones, cada una de las cuales requiere de su solución particular $T(x, y, z, t)$ de la ecuación de difusión del calor [2], [17].

El presente trabajo tiene como objetivo, estudiar las posibilidades y limitaciones de un método noestacionario, que utiliza como fuente de calor la radiación luminosa. En este método, que es una nueva variante que se propone, la luz se hace incidir homogéneamente sobre una de las superficies extremas de un cilindro, que al estar al vacío no presentaría transferencia de calor por convección, igualmente la radiación es despreciable pues en el estado noestacionario la temperatura apenas varía; manteniéndose constante la temperatura en el otro extremo.

Existen varios modelos teóricos como el de Hamilton y Crosser (HC) que predicen el comportamiento de la conductividad térmica de los compuestos y mezclas según varía la proporción de los componentes que lo forman [15]. Sin embargo, y a pesar de que estos han resistido satisfactoriamente múltiples comprobaciones experimentales, en los últimos años ha surgido una gran discusión en cuanto a la validez de los mismos cuando se trata de los nanofluidos [3,7].

La polémica, aún no resuelta, sobre la validez o no del modelo de $\mathrm{HC}$, así como de otros equivalentes [5], surge porque las mediciones de conductividad térmica que se han realizado sobre estos sistemas revelan valores que superan con creces los que predicen dichos modelos $[4,6]$. Hasta el presente, existe un número insuficiente de datos experimentales y la gran mayoría de estos se han obtenido utilizando un único método, el método del alambre caliente $[8,12]$. A su vez, otras mediciones realizadas que difieren en el método, arrojan resultados contradictorios con los reportados inicialmente [13].

En conclusión, se puede inferir que: o bien se necesita elaborar una nueva teoría o los valores de conductividad térmica que se reportan no reflejan la realidad. Esto último quizás como consecuencia de diversos factores que no se han tenido en cuenta en el momento de realizar e interpretar las mediciones.

\section{Materiales y métodos}

El método experimental que se propone consiste en lo siguiente (Figura 1a y 1b): Si el material a estudiar se trata de un sólido metálico homogéneo y transversalmente isótropo, entonces se debe preparar, con el mismo, una barra cilíndrica. Esta barra se coloca en el interior de una cámara de vacío con el propósito de impedir el intercambio de calor, por conducción y convección, a través de sus superficies frontal y lateral. La superficie trasera de la barra se fija a la pared de la cámara con la finalidad de que, en esa superficie, la temperatura se mantenga constante. Esto se lograría con una base grande preferiblemente de cobre.

En caso de que la muestra se trate de un fluido, se debe preparar un recipiente cilíndrico, en cuyo extremo frontal y en contacto directo con la muestra se fija una lámina de aluminio u otro material sólido, buen conductor del calor, sobre la cual incidirá la luz.

El otro extremo de dicho recipiente se fijará, al igual que para el caso de muestras sólidas, a la pared de la cámara de vacío, que en este caso se le practicará una abertura para permitir la entrada del fluido.

\subsection{Modelo teórico}

\subsubsection{Planteamiento del problema}

El calor siempre se propaga desde las zonas que se encuentran a mayor temperatura a las zonas donde esta es menor. Se distinguen tres formas de propagación del calor: la conducción, la convección y la radiación. 


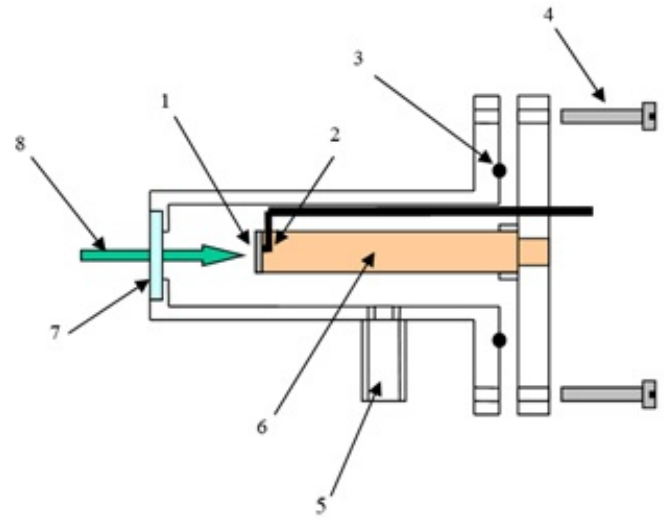

Figura 1a. La figura representa un esquema del dispositivo que se propone para medir la conductividad y difusividad térmicas de sólidos. 1. Superficie frontal. 2. Termopar. 3. O-Ring. 4. Tornillos. 5. Entrada de vacío. 6. Muestra. 7. Ventana de vidrio. 8. Luz (no modulada).

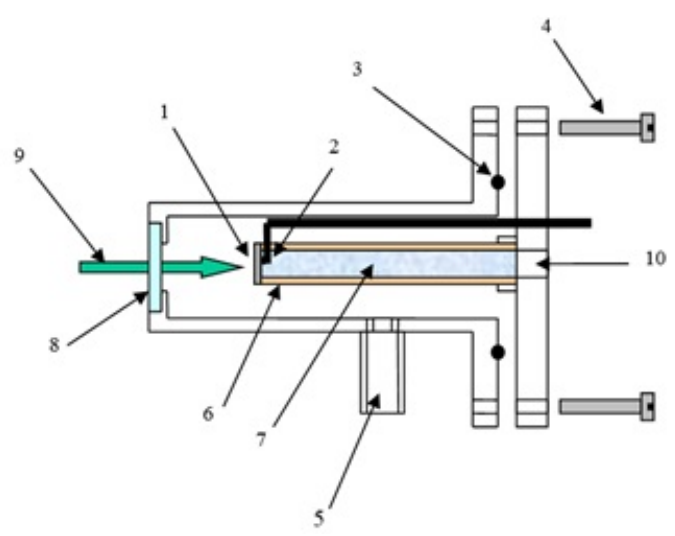

Figura 1b. La figura representa un esquema del dispositivo que se propone para medir la conductividad y difusividad térmicas de fluidos. 1. Lámina de Al. 2. Termopar. 3. O-Ring. 4. Tornillos. 5. Entrada de vacío. 6. Portamuestra. 7. Muestra. 8. Ventana de vidrio. 9. Luz (no modulada). 10. Abertura para introducir la muestra.

Se toma como modelo (Figura 2), una barra sólida homogénea cilíndrica con una longitud $l$ y área de su sección transversal $A$, por cuyas superficies frontal $x=l$ y lateral es imposible intercambiar calor por conducción o convección, mientras que la superficie trasera $x=0$ se mantiene a una temperatura $\Omega_{0}$ constante, la cual coincide con la temperatura inicial de toda la barra. Se supone que la fuente de calor de potencia $q$, está distribuida homogéneamente sobre la superficie frontal, encima de la cual incide la radiación luminosa; y que las pérdidas de calor por radiación son despreciables. En estas condiciones se puede considerar que estamos en presencia de un problema unidimensional y, por lo tanto, plantear la ecuación de difusión del calor en una sola dirección.

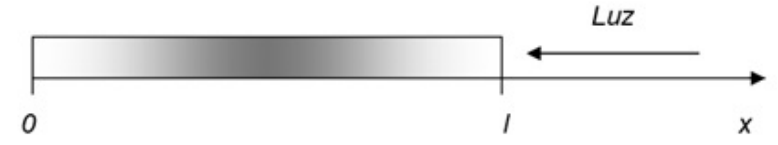

Figura 2. Barra sólida de forma cilíndrica con paredes laterales adiabáticas.

$$
\frac{\partial \Omega(x, t)}{\partial t}=\alpha \frac{\partial^{2} \Omega(x, t)}{\partial x^{2}}+F(x, t)
$$

En (1) $\alpha$ es la difusividad térmica, que viene dada por $\alpha=\frac{\chi}{\rho c}$, donde $\rho$ es la densidad, $c$ el calor específico, $F(x, t)$ tiene en cuenta las fuentes internas de calor y es igual a $F(x, t)=\frac{Q(x, t)}{\rho c}$, donde $Q(x, t)$ es la densidad de fuentes internas de calor.

En este modelo no se contempla fuentes internas de calor, ya que el mismo se genera homogéneamente sobre la superficie. Por lo tanto, la ecuación (1) a resolver se reduce a:

$$
\frac{\partial \Omega(x, t)}{\partial t}=\alpha \frac{\partial^{2} \Omega(x, t)}{\partial x^{2}}
$$

Cuyas condiciones iniciales y de fronteras son las siguientes:

$$
\begin{gathered}
\Omega(x, 0)=\Omega_{0} \\
\Omega(0, t)=\Omega_{0}, \forall 0<x<l, t>0 \\
\frac{\partial \Omega(l, t)}{\partial x}=Q
\end{gathered}
$$

donde: $Q=\frac{q}{\chi A}$, y $q$ es el flujo de calor a través de la superficie frontal de la muestra, que se considera constante ( $q$ depende directamente de la intensidad de la luz proveniente de la fuente de iluminación), $\chi$ es la conductividad térmica y $A$, el área de la sección transversal de la muestra. La solución de este problema se propone como:

$$
\Omega(x, t)=\nu(x, t)+p(x)
$$

Sustituyendo (4) en la ecuación (2) se obtiene la siguiente expresión:

$$
\frac{\partial \nu(x, t)}{\partial t}=\alpha \frac{\partial^{2} \nu(x, t)}{\partial x^{2}}+\alpha \frac{d^{2} p(x)}{d x^{2}}
$$

Esta conduce a dos nuevas ecuaciones, la primera para $p(x)$ y la segunda para $\nu(x, t)$.

$$
\frac{d^{2} p(x)}{d x^{2}}=0
$$

Con condiciones de frontera:

$$
\begin{gathered}
\frac{d p}{d x}(l)=Q \\
p(0)=\Omega_{0}
\end{gathered}
$$

La ecuación (6) es una ecuación diferencial ordinaria con coeficientes constantes, cuya solución se puede obtener en la forma: 


$$
p(x)=Q_{x}+\Omega_{0}
$$

Para $\nu(x, t)$ se tiene:

$$
\frac{\partial \nu(x, t)}{\partial t}=\alpha \frac{\partial^{2} \nu(x, t)}{\partial x^{2}}
$$

Con condiciones de frontera:

$$
\begin{aligned}
\nu(0, t) & =0 \\
\frac{\partial \nu(l, t)}{\partial x} & =0
\end{aligned}
$$

Por el método de separación de variables, en derivadas parciales, se busca la solución de esta ecuación en la forma:

$$
\begin{gathered}
\nu(x, t)=U(t) \cdot X(x) \neq 0 \\
\frac{d U(t)}{d t}+\alpha \lambda U(t)=0
\end{gathered}
$$

La ecuación (11.1) es una ecuación diferencial ordinaria y su solución es:

$$
U(t)=C \cdot e^{-\lambda \cdot \alpha \cdot t}
$$

donde: $C$ es una constante arbitraria. Para la parte espacial se obtiene:

$$
\frac{d^{2} X}{d x^{2}}+\lambda X(x)=0
$$

Cuyas condiciones iniciales y de frontera son:

$$
\begin{gathered}
X(0)=0 \\
\frac{d X}{d x}(l)=0, \forall 0<x<l \\
X(x) \neq 0
\end{gathered}
$$

La solución general de este problema queda:

$$
X(x)=D \operatorname{sen}(\sqrt{\lambda} x)+B \cos (\sqrt{\lambda} x)
$$

Exigiendo que (11.5) cumpla con (11.4) tendremos, $B=0$ y los autovalores $\lambda_{n}=\left[(2 n+1) \frac{\pi}{2 l}\right]^{2}$, $n=0,1,2,3, \ldots$. Por lo tanto, la solución de la ecuación (11.3) será:

$$
X_{n}(x)=D \operatorname{sen}\left[(2 n+1) \frac{\pi}{2 l}\right]
$$

donde: $D$ es una constante arbitraria, de aquí que la solución general de la ecuación (9) sin considerar las condiciones iniciales pueda escribirse como:

$$
\begin{aligned}
& \nu(x, t)=\sum_{n=0}^{\infty} C_{n} \cdot e^{-\left[\frac{(2 n+1) \pi}{2 l}\right]^{2} \alpha t} . \\
& \cdot \operatorname{sen}\left[\frac{(2 n+1) \pi x}{2 l}\right]
\end{aligned}
$$

La constante $C_{n}$ se determina imponiendo a la función (12) el cumplimiento de la condición inicial:

$$
\begin{aligned}
\nu(x, 0) & =\sum_{n=0}^{\infty} C_{n} \operatorname{sen}\left[\frac{(2 n+1) \pi x}{2 l}\right] \\
& =\Omega_{0}-p(x)=\varphi(x)
\end{aligned}
$$

de donde queda:

$$
C_{n} \equiv \varphi_{n}=\frac{2}{l} \int_{0}^{l} \varphi(\xi) \operatorname{sen}\left[(2 n+l) \frac{\pi \xi}{2 l}\right] d \xi
$$

Sustituyendo (14) en (12) obtenemos:

$$
\begin{aligned}
\nu(x, t)=\sum_{n=0}^{\infty} \frac{2}{l} & \int_{0}^{l} \varphi(\xi) \operatorname{sen}\left[\frac{(2 n+l) \pi \xi}{2 l}\right] d \xi \\
\cdot & e^{-\left[\frac{(2 n+1) \pi}{2 l}\right]^{2} \alpha t} \cdot \operatorname{sen}\left[\frac{(2 n+1) \pi x}{2 l}\right]
\end{aligned}
$$

Introduciendo la notación:

$$
\begin{array}{r}
G(x, \xi, t)=\sum_{n=0}^{\infty} \frac{2}{l} \operatorname{sen}\left[\frac{(2 n+l) \pi \xi}{2 l}\right] . \\
\cdot e^{-\left[\frac{(2 n+1) \pi}{2 l}\right]^{2} \alpha t} \cdot \\
\cdot \operatorname{sen}\left[\frac{(2 n+1) \pi x}{2 l}\right]
\end{array}
$$

La solución (15) toma forma:

$$
\nu(x, t)=\int_{0}^{l} G(x, \xi, t) \cdot \varphi(\xi) d \xi
$$

La función (16) es la función de Green del problema. Si integramos, queda:

$$
\begin{gathered}
\nu(x, t)=\frac{-8 l Q}{\pi^{2}} \sum_{n=0}^{\infty} \frac{(-1)^{n}}{(2 n+1)^{2}} \cdot e^{-\left[\frac{(2 n+1) \pi}{2 l}\right]^{2} \alpha t} . \\
\cdot \operatorname{sen}\left[\frac{(2 n+1) \pi x}{2 l}\right]
\end{gathered}
$$

Teniendo en cuenta (4) se llega finalmente a:

$$
\begin{aligned}
& \Omega(x, t)=\Omega_{0}+Q x-\frac{8 l Q}{\pi^{2}} \sum_{n=0}^{\infty} \frac{(-1)^{n}}{(2 n+1)^{2}} \cdot \\
& \cdot e^{-\left[\frac{(2 n+1) \pi}{2 l}\right]^{2} \alpha t} \cdot \\
& \cdot \operatorname{sen}\left[\frac{(2 n+1) \pi x}{2 l}\right]
\end{aligned}
$$


Esta expresión puede escribirse como:

$$
\begin{array}{r}
T(x, t)=T_{e s}\left\{\frac{x}{l}-\frac{8}{\pi^{2}} \sum_{n=0}^{\infty} \frac{(-1)^{n}}{(2 n+1)^{2}} \cdot e^{-\frac{t}{\tau}} .\right. \\
\left.\cdot \operatorname{sen}\left[\frac{(2 n+1) \pi x}{2 l}\right]\right\}
\end{array}
$$

donde:

$$
T_{e s}=\frac{q l}{\chi A}
$$

es la temperatura correspondiente al estado estacionario en la posición $x=l$, mientras que

$$
\tau=\frac{1}{\left[\frac{(2 n+1) \pi}{2 l}\right]^{2} \alpha}
$$

es el tiempo durante el cual la temperatura $T(x, t)$ alcanza el valor:

$$
\begin{array}{r}
T(x, t)=T_{e s}\left\{\frac{x}{l}-\frac{8}{\pi^{2}} \sum_{n=0}^{\infty} \frac{(-1)^{n}}{(2 n+1)^{2}} \cdot e^{-1} .\right. \\
\left.\cdot \operatorname{sen}\left[\frac{(2 n+1) \pi x}{2 l}\right]\right\}
\end{array}
$$

La expresión (20) representa el incremento de temperatura con relación a la temperatura inicial mientras se genera calor en la superficie de la muestra.

\subsection{Aplicabilidad del modelo para fluidos}

Si bien la ecuación de difusión del calor (1), en general, no es válida para el caso de fluidos, la misma puede aplicarse a estos, cuando sea despreciable el fenómeno de convección en el interior de ellos.

Por otra parte, los fluidos deben estar contenidos en algún recipiente, que en nuestro caso es el portamuestras. Esto provoca que los valores de difusividad y conductividad térmicas que aparecen en la expresión (20) y, que pueden ser medidos en el experimento, sean en realidad los valores efectivos del sistema compuesto por el portamuestras más el fluido. $\mathrm{O}$ sea, constituyen magnitudes que tienen en cuenta las características, tanto de uno como del otro.

Así, para la conductividad y difusividad térmicas efectivas, se tiene las expresiones siguientes [14], [18]:

$$
\begin{array}{r}
\chi_{e f}=\mu \cdot \chi_{f}+(1-\mu) \cdot \chi_{p} \\
\alpha_{e f}=\frac{\mu \cdot \chi_{f}+(1-\mu) \cdot \chi_{p}}{\mu \cdot \rho c_{f}+(1-\mu) \cdot \rho c_{p}}
\end{array}
$$

donde: $\mu$ es la proporción de área de sección transversal que, aporta el portamuestras al sistema, $\rho$ es la densidad de cada material y los subíndices $f$ y $p$ representan al fluido y al portamuestras respectivamente.

\subsection{Variación temporal de la temperatura}

La Figura 3 muestra gráficamente el comportamiento de la temperatura en la superficie frontal de una muestra líquida, como indica la figura 1b, de agua destilada. En este gráfico podemos observar, que mientras la luz incide sobre la muestra, la temperatura asciende hasta alcanzar un valor constante. A partir de ese momento, se entra en un régimen estacionario y la temperatura ya no aumenta, alcanzando el valor dado por (21); de ahí vemos que la temperatura de saturación $T_{e s}$ depende de la potencia de la fuente de calor en la superficie, la longitud de la muestra, el área de su sección transversal y de la conductividad térmica de la misma.

En la Figura 3 se observa que la temperatura de saturación de la muestra alcanza un valor de alrededor de 28 ○C. En la práctica es necesario limitar el ascenso de la temperatura, pues por una parte las propiedades térmicas de la muestra variarían durante las mediciones; y por otra se harían importantes las pérdidas de calor por el mecanismo de radiación, lo cual implicaría que el comportamiento real del sistema no se corresponda con lo que predice el modelo teórico. Por este motivo, en dependencia de los valores de conductividad térmica de la muestra, es necesario elegir adecuadamente los valores de las otras tres magnitudes involucradas en la expresión (21).

Esta elección debe efectuarse teniendo en cuenta que el tiempo durante el cual se mide, debe ser suficiente como para obtener una cantidad de puntos experimentales que permita la posterior determinación de las magnitudes buscadas.

\section{Resultados y discusión}

\subsection{Resolución del método}

En la Figura 4 se muestra, gráficamente, la diferencia de temperatura en función del tiempo $\Delta T(t)$ cuando varía el valor de la conductividad térmica en un $5 \%$. Mientras que en la Figura 5 se muestra cómo varía la temperatura $T(t)$ en función del tiempo para dos muestras cuyas conductividades térmicas se diferencian entre sí en un $5 \%$.

Para una variación determinada de la conductividad térmica, a mayor tiempo, mayores diferencias de temperaturas. O sea, la resolución del método se incrementa, por lo que será posible detectar menores diferencias de conductividad térmica utilizando un mismo sensor de temperatura.

Sin embargo, en dependencia del valor de la temperatura $T_{e s}$, así será el tiempo durante el cual es posible medir, teniendo en cuenta que, la temperatura de la muestra, por las razones antes expuestas, no debe exceder en más de unos pocos grados $\left(5-10^{\circ} \mathrm{C}\right)$ a 
la inicial y, por otra parte, se debe medir durante un tiempo superior al necesario para que la diferencia de temperatura supere aquella que es posible detectar con el sensor de temperatura disponible.

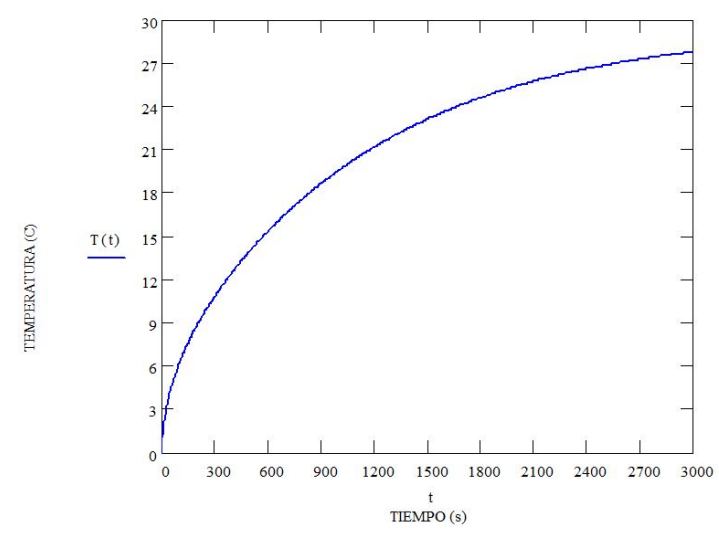

Figura 3. Dependencia temporal de la temperatura en la superficie frontal $(x=l)$, para valores de $q=0.1 \mathrm{~W}$, $l=0.02 \mathrm{~m}, A=1.131 \times 10^{-4} \mathrm{~m}^{2}, \chi=0.599 \mathrm{Wm}^{-1 \circ} \mathrm{C}^{-1}$, $\alpha=1.43 \times 10^{-7} \mathrm{~m}^{2} \mathrm{~s}^{-1}$ correspondientes a una muestra líquida de agua destilada.

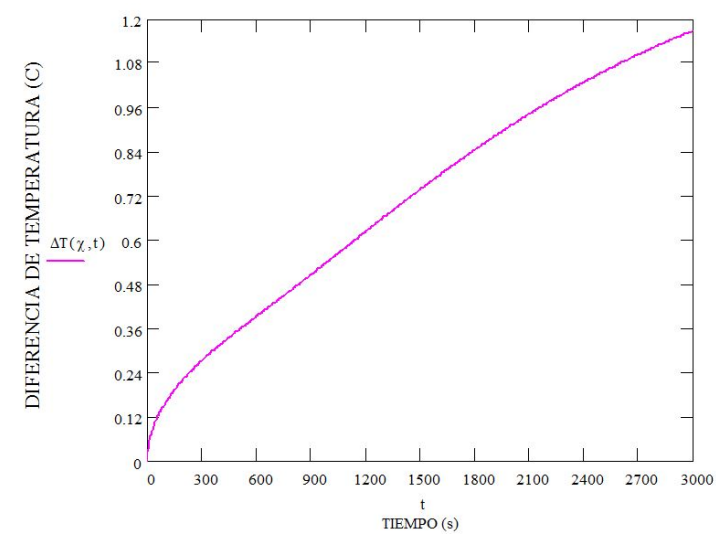

Figura 4. Diferencia de temperatura en función del tiempo para valores de $q=0.1 \mathrm{~W}, l=0.02 \mathrm{~m}, A=1.131 \times 10^{-4} \mathrm{~m}^{2}$, $\chi=0.599 \mathrm{Wm}^{-1 \circ} \mathrm{C}^{-1}, \alpha=1.43 \times 10^{-7} \mathrm{~m}^{2} \mathrm{~s}^{-1}$, y diferencia en la conductividad térmica de un $5 \%$ con respecto al valor de $0.599 \mathrm{Wm}^{-1 \circ} \mathrm{C}^{-1}$.

Por tanto, los límites del intervalo de tiempo útil para la medición, y con ello la resolución del método, están determinados, por la propia resolución del sensor de temperatura y por el valor de $T_{e s}$.

De lo dicho se deduce que: la elección del sensor de temperatura, así como de las magnitudes que intervienen en la expresión (21) depende de las diferencias mínimas de conductividad o difusividad térmicas que se deseen detectar.

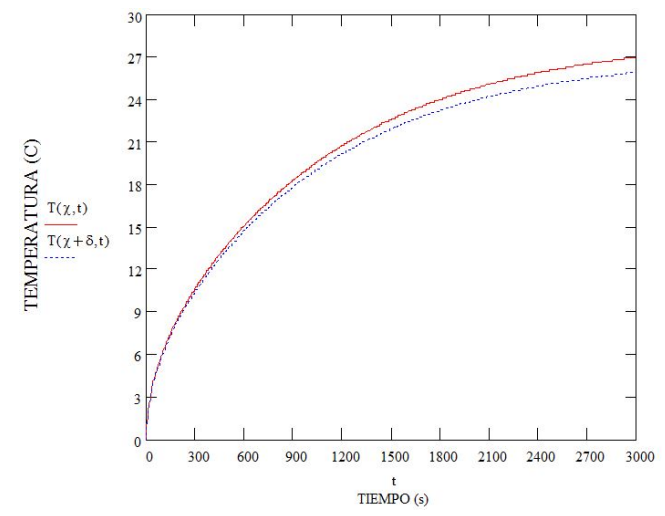

Figura 5. Dependencia temporal de la temperatura en la superficie frontal $(x=l)$ para dos muestras, cuyas conductividades térmicas se diferencian entre sí en un $5 \%$. Datos teóricos.

\subsection{Modelo HC}

El modelo de Hamilton y Crosser [15], al igual que el de Maxwell [16] y otros, predice los valores de conductividad térmica efectiva de un medio en cuyo seno se encuentran partículas diseminadas de manera aleatoria y homogénea, las cuales se encuentran suficientemente alejadas las unas de las otras, de manera que sea posible despreciar las interacciones entre ellas. Según este modelo, la conductividad térmica efectiva viene expresada por:

$$
\chi_{f}=\chi_{f b}\left(\frac{\psi+(\nu-1)-(\nu-1) \cdot(1-\psi) \cdot \lambda}{\psi+(\nu-1)+(1-\psi) \cdot \lambda}\right)
$$

donde $\psi=\frac{\chi_{n}}{\chi_{f b}}$, es la relación entre las conductividades térmicas de las partículas y el medio respectivamente, $\lambda$ es la fracción volumétrica que ocupan las partículas en el fluido, $\nu$ es un factor de forma, que tiene en cuenta la geometría de las partículas. Los subíndices $n$ y $f b$ representan a las partículas y al medio respectivamente.

Teniendo en cuenta la expresión (25) y que la capacidad calorífica específica efectiva viene expresada por:

$$
\rho c_{f}=(1-\lambda) \cdot \rho c_{f b}+\lambda \cdot \rho c_{n}
$$

Se obtiene para la difusividad térmica la siguiente relación:

$$
\alpha_{f}=\frac{\chi_{f b}\left(\frac{\psi+(\nu-1)-(\nu-1) \cdot(1-\psi) \cdot \lambda}{\psi+(\nu-1)+(1-\psi) \cdot \lambda}\right)}{(1-\lambda) \cdot \rho c_{f b}+\lambda \cdot \rho c_{n}}
$$

Si el medio en cuyo seno se encuentran las partículas es un fluido, entonces las expresiones para la conductividad y difusividad térmicas que aparecen en (20) serán las que siguen: 


$$
\begin{aligned}
\chi_{e f}= & \mu \cdot \chi_{f b}\left(\frac{\psi+(\nu-1)-(\nu-1) \cdot(1-\psi) \cdot \lambda}{\psi+(\nu-1)+(1-\psi) \cdot \lambda}\right)+ \\
& +(1-\mu) \cdot \chi_{p} \\
\alpha_{e f}= & \frac{\mu \cdot \chi_{f b}\left(\frac{\psi+(\nu-1)-(\nu-1) \cdot(1-\psi) \cdot \lambda}{\psi+(\nu-1)+(1-\psi) \cdot \lambda}\right)+(1-\mu) \cdot \chi_{p}}{\mu \cdot\left[(1-\lambda) \cdot \rho c_{f b}+\lambda \cdot \rho c_{n}\right]+(1-\mu) \cdot \rho c_{p}}
\end{aligned}
$$

donde el subíndice $\rho$ representa el portamuestras. Las Figuras 6, 7, 8 y 9 muestran las variaciones relativas de la conductividad y difusividad térmicas en función de la fracción volumétrica según (28) y (29) respectivamente.

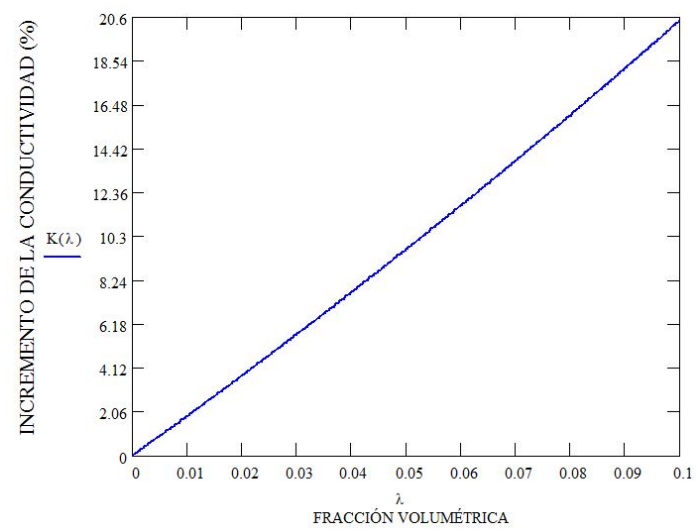

Figura 6. Incremento de la conductividad térmica vs. fracción volumétrica.

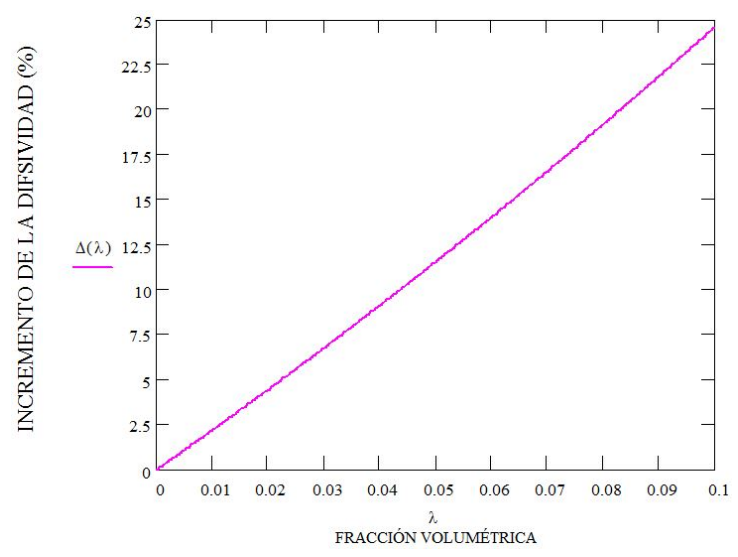

Figura 7. Incremento de la difusividad térmica vs. fracción volumétrica

En la Figura 8 se observa la diferencia de temperatura en función del tiempo $\Delta T(t)$ cuando varía la fracción volumétrica de aluminio en agua en el $1 \%$, suponiendo que, tenemos en calidad de muestra, agua destilada con nanopartículas de aluminio inmersas en $\mathrm{su}$ interior, siendo el portamuestras de vidrio.

Para ellos los valores de conductividad térmica y capacidad calorífica específica son los siguientes: $\chi_{n}=211.015 W^{\circ} C^{-1} m^{-1}, \chi_{f b}=0.599 W^{\circ} C^{-1} m^{-1}$,

$\chi_{p}=0.837 W^{\circ} C^{-1} m^{-1}, \rho_{c n}=2.453 \times 10^{6} J^{\circ} C^{-1} m^{-3}$, $\rho c_{f b}=2.187 \times 10^{6} \mathrm{~J}^{\circ} \mathrm{C}^{-1} \mathrm{~m}^{-3}$,

$\rho c_{p}=1.998 \times 10^{6} \mathrm{~J}^{\circ} \mathrm{C}^{-1} \mathrm{~m}^{-3}$.

Por su parte la Figura 9 representa, para una muestra similar, el comportamiento de la temperatura a medida que transcurre el tiempo $T(t)$.

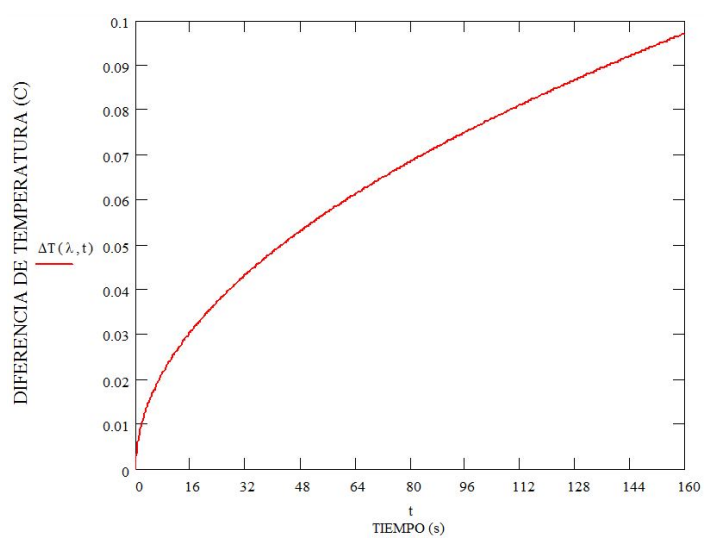

Figura 8. Diferencia de temperatura en función del tiempo, para un incremento de la fracción volumétrica en un $1 \%$.

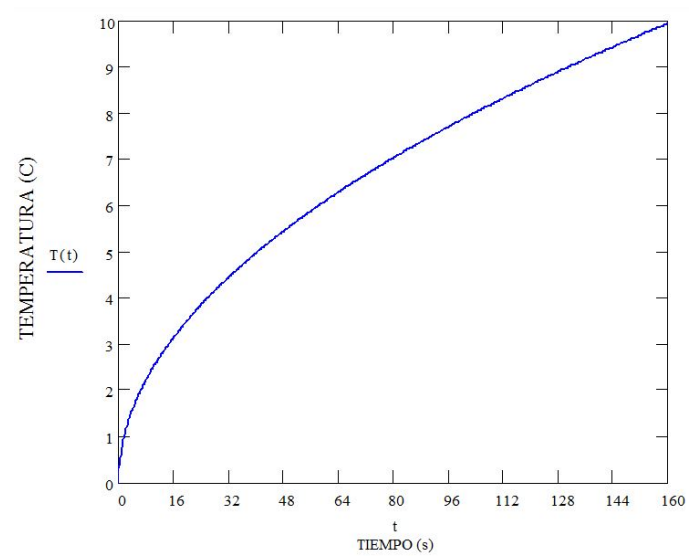

Figura 9. Temperatura en función del tiempo. Suponiendo que tenemos en calidad de muestra agua con partículas de aluminio inmersas en su interior y que el material del portamuestras es de vidrio.

Para comprobar la validez o no del modelo de HC, basta con medir los valores de difusividad térmica de varias muestras que se diferencien entre sí solamente en el valor de su fracción volumétrica y luego verificar que la expresión (29) se ajusta suficientemente bien a los puntos experimentales resultantes de las mediciones. Si se desea estudiar el comportamiento de un nanofluido, entonces se procede de la forma antes expuesta. Para realizar este tipo de estudio empleando el método que se propone, es necesario elegir el sensor de temperaturas adecuadamente, porque la resolución del 
mismo debe estar en concordancia con las diferencias mínimas de conductividad o difusividad térmicas que se requieren detectar.

\section{Conclusiones}

Se resolvió la ecuación de difusión del calor para las condiciones propias del método propuesto, obteniéndose una expresión a partir de la cual es posible determinar tanto los valores de conductividad como de difusividad térmicas. La elección del sensor de temperatura, así como de las magnitudes que intervienen en la expresión (21) depende de las diferencias mínimas entre conductividades y difusividades térmicas que se deseen detectar, como ilustran las Figuras 4 y 5. En este sentido, para realizar mediciones de corto tiempo (alrededor de $5 \mathrm{~min}$ ) se necesita un sensor cuya precisión llegue hasta la centésima de grado, si fuese menos preciso habría que medir por más tiempo (aproximadamente una hora), esto aumentaría considerablemente la temperatura en la muestra, dando lugar al fenómeno de conducción del calor por radiación, lo cual no es deseado.

Para medir en materiales sólidos es necesario que estos puedan ser maquinados, de manera que sea posible preparar con ellos barras, cilíndricas o de otra geometría. El método permite comprobar el modelo de HC, siempre que se cuente con un medidor de temperaturas con una resolución tal que le permita diferenciar hasta la centésima de grado centígrado.

\section{Referencias}

[1] J. N. Fox and R. H. McMaster, "Measurement of the thermal properties of a metal using a relaxation method," American Journal of Physics, vol. 43, pp. 1083-1086, 1975.

[2] T. Ficker, "A non-stationary method for the measurement of the thermal conductivity of solids in student laboratories," European Journal of Physics, vol. 17, pp. 307-310, 1996.

[3] D. H. Hurley, R. S. Schley, M. Khafizov, and B. L. Wendt, "Local measurement of thermal conductivity and diffusivity," Review of Scientific Instruments, 2015.

[4] P. Keblinski, S. R. Phillpot, S. U. S. Xhoi, and J. A. Eastman, "Mechanisms of heat flow in suspensions of nano-sized particles (nanofluids)," International Journal of Heat and Mass Transfer, vol. 45, pp. 855-863, 2002.

[5] Q. Xue and W. M. Xu, "A model of thermal conductivity of nanofluids with interfacial shells," Materials Chemistry and Physics, vol. 90, pp. 298-301, 2005.
[6] E. Gregorová, "The thermal conductivity of alumina-water nanofluids from the viewpoint of micromechanics," Microfluidics and Nanofluidics, vol. 16, pp. 19-28, 2014.

[7] L. Q. Yimin, Xuan, "Heat transfer enhancement of nanofluids," International Journal of Heat and Fluid Flow, vol. 21, pp. 58 - 64, 2000.

[8] S. A. Putnam, D. G. Cahill, P. V. Braun, Z. Ge, and R. G. Shimmin, "Thermal conductivity of nanoparticle suspensions," Journal of Applied Physics, vol. 99, 2006.

[9] J. A. Eastmaan, S. U. S. Choi, S. Li, W. Yu, and L. J. Thompson, "Anomalously increased effective thermal conductivities of ethylene glycol-based nanofluids containing copper nanoparticles," $A p$ plied Physics Letters, vol. 78, 2001.

[10] S. U. S. Choi, Z. G. Zhang, W. Yu, F. E. Lockwood, and E. A. Grulke, "Anomalous thermal conductivity enhancement in nanotube suspensions," vol. 79, 2008.

[11] H. Xie, J. Wang, T. Xi, and Y. Liu, "Thermal conductivity of suspensions containing nanosized sic particles," International Journal of Thermophysics, vol. 23, pp. 571-580, 2002.

[12] D. Klingenberg and D. Venerus, "Thermal conductivity measurements in nanofluids," in Presentación de Curso, U. States, Ed. American Institute of Chemical Engineers, 2006.

[13] Z. Xing, G. Hua, and F. Motoo, "Effective thermal conductivity and thermal diffusivity of nanofluids containing spherical and cylindrical nanoparticles," Journal of Applied Physics, vol. 31, no. 6, pp. 593-599, 2007.

[14] E. Marín, "Escuchando la luz: Breve historia y aplicaciones del efecto fotoacústico," in Presentación de Curso, México, Ed. Seminario Repensar la Fúsica, Instituto Politécnico Nacional, 2016.

[15] R. L. Hamilton and O. K. Crosser, "Thermal conductivity of theory of heterogeneus two-componet system," Industrial \& Engineering Chemistry Fundamentals, vol. 1, pp. 187-191, 1962.

[16] J. C. Maxwell, "A treatise on electricity and magnetism," Clarendon Press, Oxford, UK 2nd ed, vol. 1, p. 435, 1881.

[17] A. N. Tijonov and Samarsky, Ecuaciones de la física matemática, p. 824, 1972.

[18] O. Delgado, "Contribución de la técnica fotoacústica a la caracterización térmica y óptica de materiales," Tesis Doctoral, Facultad de Física, Universidad de La Habana, La Habana, 2003. 